\title{
APPLICATION OF THE THERMODYNAMIC THEORY OF IRREVERSIBLE PROCESSES TO NORMAL GRAIN GROWTH
}

\author{
P.R. Rios ${ }^{1,2}$, G. Gottstein ${ }^{1}$ and L.S. Shvindlerman ${ }^{1}$ \\ ${ }^{1}$ Institut für Metallkunde und Metallphysik, RWTH Aachen, D-52056 Aachen, Germany \\ ${ }^{2}$ Universidade Federal Fluminense, Escola de Engenharia Industrial Metalúrgica de Volta Redonda, \\ Av. dos Trabalhadores, 420, Volta Redonda, RJ, 27255-125 Brasil
}

(Received July 26, 2000)

(Accepted in revised form November 7, 2000)

Keywords: Grain growth; Thermodynamics

\section{$\underline{\text { Introduction }}$}

It is well established that the driving force for grain growth is the grain boundary interfacial free energy. As grain growth takes place the total grain boundary interfacial free energy decreases with time, $t$ :

$$
\frac{d G}{d t}=\frac{d(\gamma S)}{d t}<0
$$

where $G, S$ and $\gamma$ are the Gibbs free energy, the total grain boundary interface area and the grain boundary free energy per unit of grain boundary area.

The grain boundary migration rate during grain growth is proportional to the pressure difference across the grain boundaries, $\Delta p$, or equivalently to the grain boundary curvature $(1,2)$ :

$$
\nu=M \Delta p=-M \gamma \kappa
$$

where $v$ is the grain boundary migration rate or the grain boundary velocity, $M$ is the grain boundary mobility and $\kappa$ is the grain boundary curvature. The minus sign is necessary for grain growth since the grain boundary moves towards its center of curvature.

Computer simulation of grain growth has significantly developed in the last decade (3-14). Computer simulations normally belong to one of two distinct groups. In the first group there are the computer simulations that use Eq. 2 at each point of the grain boundary as a "rule" for the grain boundary migration. An example would be the "front tracking models"(5). In the second group there are models which assume a different rule for grain boundary migration, for example, Monte Carlo Potts models $(3,4)$. These models often use Eq. 1 to establish the direction of migration. The expression "curvature driven models" is often used when referring to the first group. It is clearly relevant to understand to what extent such models are equivalent and this is connected to a more precise understanding of the relationship between Eqs. 1 and 2.

In addition to Eqs. 1 and 2 another characteristic has been found to be important for grain growth: scaling. After a transient period, grain growth evolution reaches the quasi-stationary state in which both grain size and grain shape distribution remain invariant when suitably scaled. The parabolic rate law, $d R^{2} / d t=$ constant, where $R$ is the mean grain radius is characteristic of this state. The quasi-stationary state was predicted in the early analytical theories (15-17) of grain growth and has been extensively 
confirmed by computer simulation results (3-14). Computer simulations invariably find $d R^{2} / d t=$ constant and the "quasi-stationary" grain size/shape distributions are similar even when different computer simulation methods are used $(10-13)$. The grain size distribution obtained from computer simulation (18-20) can be described by a grain size distribution obtained by an analytical model(18). However the analytical model is unable to predict the value of $d R^{2} / d t$ that has to be found from the computer simulation data.

In spite of the large amount of research on normal grain growth the relationship among Eqs. 1, 2 and the quasi-stationary state is not yet fully understood. In this work the formalism of the Thermodynamic Theory of Irreversible Processes (21) is applied to normal grain growth in order to clarify this point.

\section{Irreversible Thermodynamics}

The irreversible entropy production, $\sigma$, can be written as (21):

$$
\sigma=-\frac{1}{T} \frac{d G}{d t}
$$

From a general point of view (22) one can say that the grain boundary velocity, $v$, is proportional to a driving force $P$ :

$$
\nu=M P
$$

This driving force has the dimensions of energy per unit of volume which is conceptually equivalent to a pressure, a force acting per unit of area on a grain boundary. As a certain grain boundary area $S$ moves with velocity $v$ the rate of free energy change is given by:

$$
-\frac{d G}{d t}=S \nu P=S M P^{2}
$$

where $M$ is the grain boundary mobility.

Eqs. 4 and 5 suggest that for grain boundary migration it is convenient to use the rate of production of irreversible entropy per unit of area of interface $(23), \sigma_{\mathrm{S}}$, given by

$$
\sigma_{s}=-\frac{1}{T S} \frac{d G}{d t}
$$

where $S$ is the total grain boundary area. The rate of the irreversible entropy production can be written as a product of generalized forces and the corresponding rates or generalized "fluxes" of the irreversible processes. In the present case it is enough to consider one rate or generalized flux, $J$, and one force, $X$ (21):

$$
T \sigma_{s}=-\frac{1}{S} \frac{d G}{d t}=J X
$$

where the flux is assumed to be linearly related to the force by a linear phenomenological law (21):

$$
J=L X
$$

where $L$ is a phenomenological coefficient assumed to be constant.

From Eqs. 7,8 an useful expression can be found 


$$
-\frac{1}{S} \frac{d G}{d t}=\frac{J^{2}}{L}=L X^{2}
$$

From Eqs. 4,5 and 8,9 one can readily identify $L=M, X=P$ and consequently $J=v$. In the present work only grain growth and its associated driving force for grain boundary motion are considered but the above treatment can be applied to grain boundary migration in general.

\section{$\underline{\text { Total Grain Boundary Area vs. Grain Boundary Curvature }}$}

The irreversible entropy production can be written as a function of grain boundary area per unit of volume, $S$, by using Eqs. 1 and 6 and from the constraint that the total volume remains constant:

$$
T \sigma_{s}=-\frac{\gamma}{S} \frac{d S}{d t}=-\frac{\gamma}{S_{V}} \frac{d S_{V}}{d t}
$$

For normal grain growth the average grain boundary velocity $\langle\nu\rangle$ is a suitable choice for the reaction rate or generalized "flux." Using Eqs. 9,10:

$$
\langle\nu\rangle^{2}=-\frac{L \gamma}{S_{V}} \frac{d S_{V}}{d t}
$$

Eq. 11 relates a "local" property, the grain boundary velocity, to a "global" property the grain boundary area per unit of volume. Eq. 11 does not depend on any stereological assumptions and is valid for non-steady state grain growth.

In order to find an expression for $\langle\nu\rangle$ one can use $(24,25)$ :

$$
\langle\nu\rangle=\frac{1}{S} \frac{d V_{S}}{d t}
$$

Eq. 10 was derived for the growth of a second phase and in that case $d V_{S}$ represented the increase in the volume of the second phase. In the present case $d V_{S}$ represents the volume swept by the moving grain boundary. An approximate expression for the volume swept(26) can be found considering $N$ equal grains of mean grain volume $u_{m}$. In such an idealized situation $N-d N$ grains grow by $d u_{m}$ whereas $d N$ grains of volume $u_{m}$ disappear so that the total volume, $V_{T}=N u_{m}=(N-d N)\left(u_{m}+d u_{m}\right)$, remains constant. Clearly the volume swept due to the displacement of the boundaries is $d V_{S}=(N-d N) d u_{m}=$ $N d u_{m}$ so that using Eqs.11,12 one can write:

$$
\langle\nu\rangle=\frac{1}{S_{V}} \frac{d \ln u_{m}}{d t}=-\frac{1}{S_{V}} \frac{d \ln N_{V}}{d t}
$$

where $N_{V}=1 / u_{m}$ is the number of grains per unit of volume. As a consequence:

$$
\left(\frac{d \ln N_{V}}{d t}\right)^{2}=-\frac{L \gamma}{2} \frac{d S_{V}^{2}}{d t}
$$

Eq. 14 is valid for non-steady state grain growth. Although the final results are different, the concept of the volume swept was introduced by Rhines, Craig and DeHoff (27) and later used by Doherty (28) and Vandermeer (29).

The grain boundary area per unit of volume, $S_{V}$, and the mean grain volume, $u_{m}$, can be expressed as a function of the mean grain radius, $R$, using appropriate shape factors, $k_{S V}$ and $k_{V}$ : 


$$
\begin{gathered}
S_{V}=\frac{k_{S V}}{R} \\
u_{m}=k_{V} R^{3}
\end{gathered}
$$

During non-steady state grain growth $k_{S V}$ and $k_{V}$ depend on the grain size/shape distribution, which can change with time. For quasi-stationary normal grain growth treated here $k_{S V}$ and $k_{V}$ are constant. Inserting Eqs. 15 and 16 into Eq. 14 one obtains the parabolic law characteristic of the quasi-stationary state:

$$
\frac{d R^{2}}{d t}=\frac{2}{9} k_{S V}^{2} L \gamma
$$

In Eqs. $11,13\langle\nu\rangle$ was directly related to the decrease in the total grain boundary area per unit of volume. Alternatively, one could relate $\langle\nu\rangle$ to the average driving force that acts on the grain boundaries. By taking the average on both sides of Eq. 2 one can write:

$$
\langle\nu\rangle=M\langle\Delta p\rangle=-M \gamma\langle\kappa\rangle
$$

The work done by $\left\langle\Delta p>\right.$ as the grain boundaries sweep $d V_{S}, \delta W$, must be equal to the decrease in the total interfacial free energy:

$$
\delta W=\langle\Delta p\rangle d V_{S}=-d G=-\gamma d S
$$

as a consequence:

$$
\langle\Delta p\rangle=-\gamma\langle\kappa\rangle=-\gamma \frac{d S}{d V_{S}}
$$

It is trivial to see that the choices: $J=\langle\nu\rangle$ and $X=\langle\Delta p\rangle=-\gamma\langle\kappa\rangle$ are fully consistent:

$$
T \sigma_{S}=-\frac{\gamma}{S} \frac{d S}{d t}=-\langle\nu\rangle\langle\gamma \kappa\rangle=-\frac{\gamma}{S} \frac{d V_{S}}{d t} \frac{d S}{d V_{S}}
$$

Therefore $\langle v\rangle$ can be either directly related to the decrease in the total grain boundary area or to the average driving force that acts on the grain boundaries. It can be easily checked that the final results are the same in both cases with $L$ equal to $M$.

\section{Minimum Entropy Production and the Quasi-Stationary State}

Inserting Eq. 15 into Eq. 10 one obtains:

$$
T \sigma_{S}\left(R, k_{S V}\right)=\frac{1}{R} \frac{d R}{d t}-\frac{1}{k_{S V}} \frac{d k_{S V}}{d t}
$$

Eq. 22 shows that the entropy production can be expressed as a function of two variables: $R$ that corresponds to a length scale of the system and $k_{S V}$ that is a function of the grain size/shape distribution. The first term is always positive and continuously decreases with time as $R$ increases. It has no minimum but goes to zero as $R \rightarrow \infty$ that corresponds to the equilibrium condition. The $d k_{S V} / d t$ term on Eq. 22 vanishes when the system reaches the quasi-stationary state in which $k_{S V}$ remains constant.

According to Prigogine's Principle of Minimum Entropy Production(21) the entropy production is a minimum at the steady-state. So strictly speaking this principle is not valid for the quasi-stationary state. The quasi-stationary state is not a steady-state in the thermodynamical sense since the entropy production is not constant. 
From Eqs. 17,22, supposing that $k_{S V}$ remains constant an expression for the entropy production in the quasi-stationary state, can be written as:

$$
T \sigma_{S}\left(R, k_{S V}=\text { constant }\right)=\frac{M \gamma k_{S V}^{2}}{9 R^{2}}
$$

\section{$\underline{\text { Summary }}$}

In summary, the irreversible thermodynamics treatment, although possibly somewhat more general as the driving force need not be specified, gives a result identical to that obtained from the usual relationship of grain boundary velocity and curvature provided that the generalized flux and force are consistently defined.

\section{$\underline{\text { Acknowledgments }}$}

One of the authors (PRR) is grateful to Conselho Nacional de Desenvolvimento Científico e Tecnológico $(\mathrm{CNPq})$ of the Brazilian Government for the financial support through a Senior Scholar$\operatorname{ship}(200048 / 82-1)$.

\section{$\underline{\text { References }}$}

1. J. von Neumann, Metal Interfaces, ASM Seminar, p. 65, ASM, Materials Park, OH (1952).

2. W. W. Mullins, J. Appl. Phys. 27, 900 (1956).

3. M. P. Anderson, D. J. Srolovitz, G. S. Grest, and P. S. Sahni, Acta Metall. 32, 783 (1984).

4. M. P. Anderson, G. S. Grest, and D. J. Srolovitz, Phil. Mag. B. 59, 293 (1989).

5. H. J. Frost, C. V. Thompson, C. L. Howe, and J. Whang, Scripta Metall. 22, 65 (1988).

6. T. Nagai, S. Ohta, and K. Kawasaki, Mater. Sci. Forum. 94-96, 313 (1992).

7. K. Marthinsen, O. Hunderi, and N. Ryum, Acta Mater. 44, 1681 (1996).

8. S. P. A. Gill and A. C. F. Cocks, Acta Mater. 44, 4777 (1996).

9. D. Fan and L.-Q. Chen, Acta Mater. 45, 611 (1997).

10. D. Weygand, Y. Bréchet, and Z. Néda, Phil. Mag. B. 75, 937 (1997).

11. D. Weygand, Y. Bréchet, and J. Lépinoux, Phil. Mag. B. 78, 329 (1998).

12. V. Tikare, E. A. Holm, D. Fan, and L.-Q. Chen, Acta Mater. 47, 363 (1999).

13. W. Fayad, C. V. Thompson, and H. J. Frost, Scripta Mater. 40, 1199 (1999).

14. V. E. Fradkov and D. G. Udler, Adv. Phys. 43, 739 (1994).

15. I. M. Lifshitz and V. V. Slyozov, J. Phys. Chem. Solids USSR. 19, 315 (1961).

16. C. Wagner, Z. Elektrochem. 65, 581 (1961).

17. M. Hillert, Acta Metall. 13, 227 (1965).

18. P. R. Rios, Scripta Mater. 42, 349 (2000).

19. P. R. Rios, Scripta Mater. 41, 1283 (1999).

20. P. R. Rios, Scripta Mater. 40, 665 (1999).

21. I. Prigogine, Introduction to Thermodynamics of Irreversible Processes, 2nd ed., John Wiley, New York (1961).

22. G. Gottstein and L. S. Shvindlerman, Grain Boundary Migration in Metals, CRC Press, Boca Raton, FL (1999).

23. E. S. Machlin, Trans. AIME. 197, 437 (1953).

24. J. W. Cahn and W. Hagel, Decomposition of Austenite by Diffusional Porcesses, ed. Z. D. Zackay and H. I. Aaronson, p.131, Interscience Publishers, New York (1960).

25. R. T. DeHoff, in Simulation and Theory of Evolving Microstructures, ed. M. O. Anderson and A. D. Rollet, p. 219, The Minerals, Metals \& Materials Society, Warrendale, PA, 1990 (1960).

26. P. R. Rios, Scripta Mater. 34, 1185 (1996).

27. F. N. Rhines, K. R. Craig, and R. T. DeHoff, Metall. Trans. 5A, 413 (1974).

28. R. D. Doherty, Metall. Trans. 6A, 588 (1975).

29. R. A. Vandermeer, Acta Metall. Mater. 40, 1159 (1992). 